\title{
Atomic force microscopy characterization of an electrochemical DNA-biosensor
}

\author{
Ana-Maria Chiorcea, Ana Maria Oliveira Brett* \\ Departamento de Química, Faculdade de Ciências e Tecnologia, Universidade de Coimbra, 3004-535 Coimbra, Portugal
}

Received 23 June 2003; accepted 30 September 2003

\begin{abstract}
Electrode surface characteristics represent an important aspect on the construction of sensitive DNA electrochemical biosensors for rapid detection of DNA interaction and damage. Two different immobilization procedures of double-stranded DNA (dsDNA) at the surface of a HOPG electrode were evaluated by MAC mode AFM performed in air. A thin dsDNA adsorbed film forming a network structure with holes exposing the electrode surface and a thick dsDNA film completely covering the electrode surface, presenting a much rougher structure, were investigated. The DNA surface characteristics and structure are discussed with respect to the degree of surface coverage.
\end{abstract}

(C) 2004 Elsevier B.V. All rights reserved.

Keywords: DNA-biosensor; dsDNA; HOPG; AFM; Atomic force microscopy

\section{Introduction}

DNA electrochemical biosensors enable rapid detection of chemicals that cause irreversible damage to DNA, by monitoring the changes in the oxidation peaks of the DNA bases guanine and adenine, and have important applications in the better understanding and evaluation of DNA-drug interaction mechanisms [1-5]. A critical issue in the development of a DNA-based biosensor is the sensor material and the degree of surface coverage that influences directly the sensor response. Despite the extensive use of DNA-biosensors, there have been relatively few studies about the morphology of DNA immobilized on the carbon electrode surface.

Magnetic A/C Mode Atomic Force Microscopy (MAC Mode AFM) is a gentle technique that permits the direct visualisation of biomolecules that are softly bound to the electrode surface [6].

The DNA electrochemical biosensors used have consisted of a highly oriented pyrolytic graphite (HOPG) electrode with DNA immobilized on its surface. Two different approaches of immobilization of double-stranded DNA (dsDNA) were evaluated for constructing calf-thymus

\footnotetext{
* Corresponding author. Tel.: +351-2398-35295; fax: +351-239835295 .

E-mail address: brett@ci.uc.pt (A.M. Oliveira Brett).
}

modified electrodes. MAC mode AFM has been used to analyze the structural characteristics of the HOPG electrode modified by a thin and a thick film of dsDNA.

\section{Experimental}

Calf-thymus dsDNA (sodium salt, type I) was purchased from Sigma, Spain and was used without further purification. The electrolyte used was $\mathrm{pH} 4.5,0.1 \mathrm{M}$ acetate buffer solution and was prepared using analytical grade reagents and purified water from a Millipore Milli-Q system (conductivity $<0.1 \mu \mathrm{S}$ $\mathrm{cm}^{-1}$ ). The DNA solutions were obtained by direct dilution of the appropriate volume in acetate buffer. The concentrations of the solutions were calculated from absorbance measurements at $260 \mathrm{~nm}$. HOPG, grade $\mathrm{ZYH}$, of rectangular shape with $15 \times 15 \times 2 \mathrm{~mm}$ dimensions, from Advanced Ceramics, UK, was used throughout this study as a substrate. The HOPG was freshly cleaved with adhesive tape prior to each experiment and was imaged by AFM in order to establish its cleanliness.

The dsDNA thin film was prepared by free adsorption from $100 \mu \mathrm{l}$ of $60 \mu \mathrm{g} / \mathrm{ml}$ dsDNA solution in $\mathrm{pH} 4.50 .1 \mathrm{M}$ buffer acetate onto HOPG surface and incubated for $3 \mathrm{~min}$. The adsorption process was stopped by gently rinsing the sample with a jet of Milly Q water and the HOPG with adsorbed DNA was dried with nitrogen and imaged in air. 
The thick dsDNA film was prepared by covering the HOPG electrode surface area with $100 \mu \mathrm{l}$ solution prepared by dissolving $3 \mathrm{mg}$ of dsDNA in $80 \mu \mathrm{l} \mathrm{pH} 4.50 .1 \mathrm{M}$ acetate buffer electrolyte solution, and leaving the electrode in sterile atmosphere to dry overnight.

AFM was performed with a Pico SPM controlled by a MAC Mode module and interfaced with a PicoScan controller from Molecular Imaging, USA. All the AFM experiments were performed with a CS AFM S scanner with a scan range $6 \mu \mathrm{m}$ in $x-y$ and $2 \mu \mathrm{m}$ in $z$, Molecular Imaging Silicon type II MAClevers $225 \mu \mathrm{m}$ length, $2.8 \mathrm{~N} / \mathrm{m}$ spring constant and 60-90 kHz resonant frequencies (Molecular Imaging) were used. All images were taken at room temperature, scan rates $1.0-1.3$ lines $\mathrm{s}^{-1}$. The images were processed by flattening in order to remove the background slope and the contrast and brightness were adjusted. All images were visualised in three dimensions using the Scanning Probe Image Processor, SPIP, and version 2.3011, Image Metrology ApS, Denmark. Section analyses over dsDNA films as well as RMS roughness measurements were performed with PicoScan software version 6.0, Molecular Imaging.
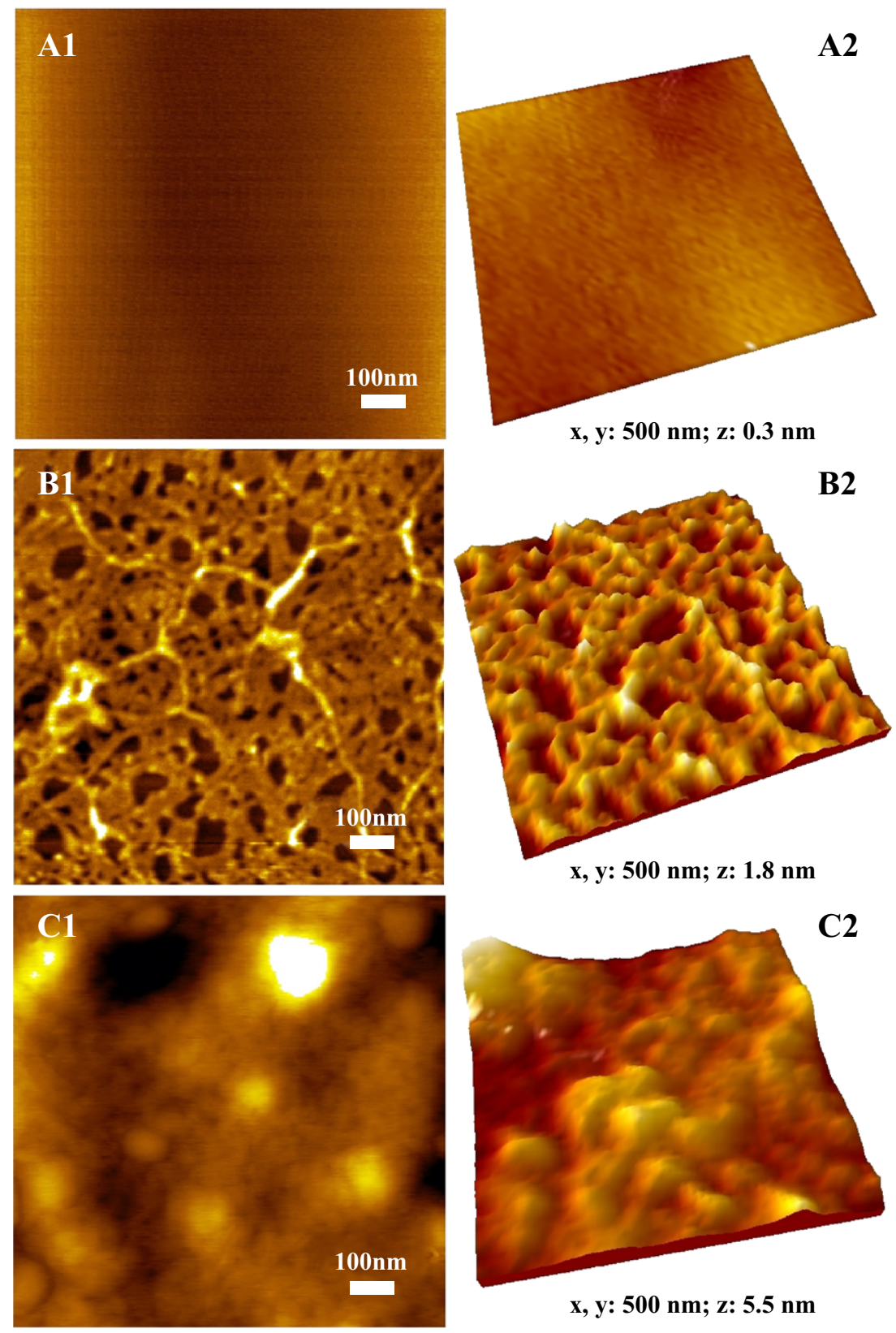

$\mathrm{x}, \mathrm{y}: 500 \mathrm{~nm} ; \mathrm{z}: 5.5 \mathrm{~nm}$

Fig. 1. MAC Mode AFM topographical images in air of: (A1 and A2) clean HOPG electrode surface; (B1 and B2) thin film dsDNA-biosensor surface, prepared onto HOPG by $3 \mathrm{~min}$ free adsorption from a solution of $60 \mu \mathrm{g} / \mathrm{ml}$ dsDNA in pH $4.50 .1 \mathrm{M}$ acetate buffer electrolyte; (C1 and C2) thick film dsDNAbiosensor surface, prepared onto HOPG by evaporation from solution of $37.5 \mu \mathrm{g} / \mathrm{ml} \mathrm{dsDNA}$ in $\mathrm{pH} 4.50 .1 \mathrm{M}$ acetate buffer electrolyte; (A1, B1 and C1) twodimensional view $1 \times 1 \mu \mathrm{m}$ scan size and (A2, B2 and C2) three-dimensional view $500 \times 500 \mathrm{~nm}$ scan size. 


\section{Results and discussion}

In Fig. 1.A1 and A2 are presented typical AFM images in air of the freshly cleaved HOPG substrate utilised during the experiments. The HOPG surface is extremely smooth, presenting a RMS roughness of less than $0.06 \mathrm{~nm}$, as calculated from a typical $1000 \times 1000 \mathrm{~nm}$ scan size AFM image in air, Fig. 1.A1, which enables the identification of the topography changes when the surface is modified with dsDNA.

The electrochemical studies performed with DNA-electrochemical biosensors show an increased biosensor sensitivity when prepared from a $\mathrm{pH} 4.5$ dsDNA solution $[3,4]$. For this reason, $\mathrm{pH} 4.5$ has been chosen for performing the AFM studies.

\subsection{Immobilization of a thin layer of $d s D N A$}

The HOPG electrode was modified by a thin dsDNA film obtained by free adsorption and was characterized by MAC Mode AFM in air. The dsDNA adsorbed spontaneously onto the HOPG electrode surface, forming a non-uniform thin layer of dsDNA, Fig. 1.B1 and B2. Since the bases at both ends of the dsDNA molecules are not hydrogen-bonded to their complement due to a continuously association-dissociation [7], they are free to interact with the hydrophobic HOPG surface through hydrophobic interactions. The attachment of the dsDNA ends is followed by the adsorption of the middle sections of the molecules [8]. The thin dsDNA film has a network structure, with holes that left many HOPG uncovered areas, revealing the flat HOPG surface underneath. Section analysis over the holes allows the determination of the film thickness of $0.94 \pm 0.2 \mathrm{~nm}$. Many overlapped molecules were also observed all over the images, Fig. 1.B2. After dsDNA network modification, the HOPG surface presented a RMS roughness of $0.36 \mathrm{~nm}$ for the same scan size of $1000 \times 1000 \mathrm{~nm}$, Fig. 1.B1.

\subsection{Immobilization of a thick layer of $d s D N A$}

A thick film of dsDNA was prepared onto HOPG surface by evaporation of a certain volume of a dsDNA solution, using the immobilization procedure described in the experimental section, by a similar method to that used with dsDNA on a glassy carbon electrode $[3,4]$. A thick film of dsDNA visible to the eye covered the electrode surface. MAC mode AFM images in air of the HOPG surface showed the HOPG surface completely covered by a uniform multilayer film of dsDNA, Fig. 1.C1 and C2. The topography of the film shows dsDNA nuclei of many different sizes from 3 to $50 \mathrm{~nm}$ height, and 50 to $400 \mathrm{~nm}$ diameter measured at half height. The calculated RMS roughness, Fig. 1.C1, was 3.41 $\mathrm{nm}$, demonstrating a much rougher topography of the modified electrode.

\subsection{Comparison between thin and thick layer of DsDNA}

The dsDNA modified electrodes can be used to probe and study the mechanism of interaction of compounds with dsDNA $[3,4]$.

The dsDNA networks formed at the HOPG electrode during the formation of thin dsDNA layers define different active surface areas on the DNA electrochemical biosensor. Due to the existence of pores on the DNA structure, areas of the HOPG surface are not covered by the molecular film, Fig. $2 \mathrm{~A}$. These uncovered regions may act as a system of microelectrodes with nanometer or micrometer dimensions. The two dimensional dsDNA networks form a biomaterial matrix to attach and study other molecules such as chemical drugs.

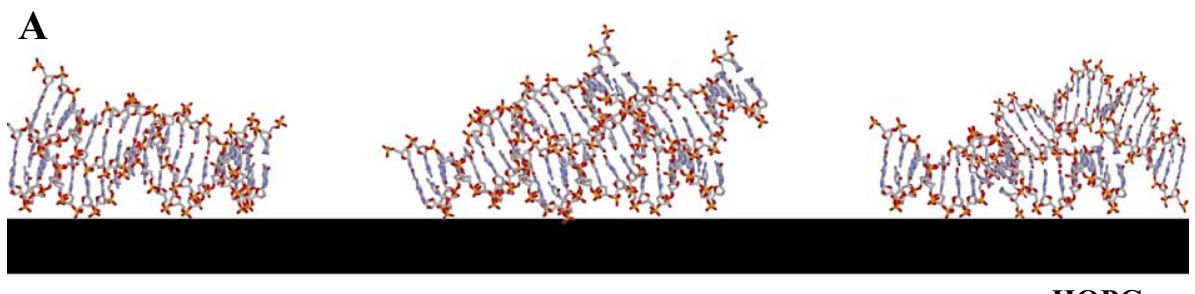

HOPG

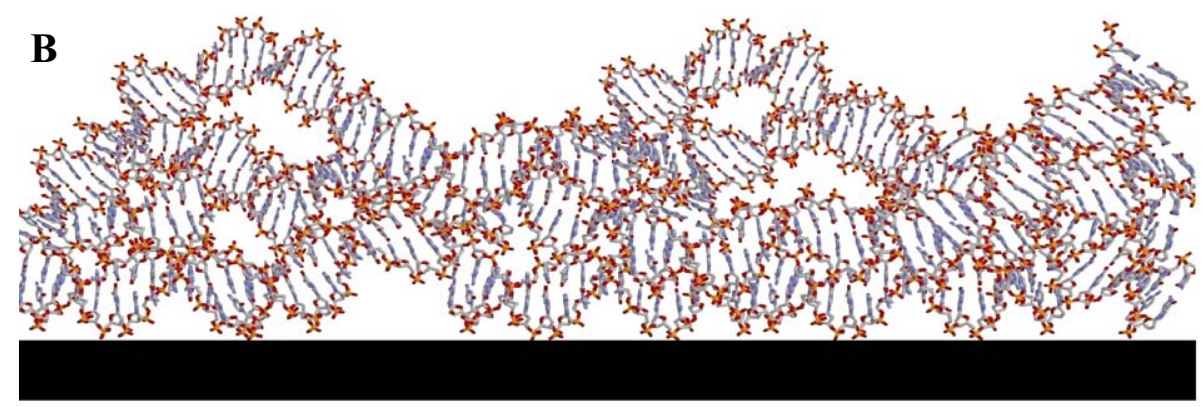

HOPG

Fig. 2. Schematic models of the adsorption of dsDNA at the HOPG electrode surface, with the formation of: (A) thin and (B) thick dsDNA films. 
The dsDNA modifications due to the interaction with substances are generally detected by changes of the electrochemical behavior of the immobilized dsDNA, specifically the purinic bases [3,4]. However, some specific drugs that damage DNA are strongly adsorbed on the carbon electrode surface and also have electrochemical activity. The major problem encountered with the HOPG electrodes modified by a thin film of dsDNA is the fact that the electrode is not completely covered allowing the diffusion of drug molecules from the bulk solution to the surface and their nonspecific adsorption. This leads to two contributions to the electrochemical signal, one from the simple adsorbed drug compound and other from the damage to immobilized dsDNA, and it is difficult to distinguish between the two signals [4].

The big advantage of dsDNA modified by a thick film is that the undesired binding of drug molecules to the electrode surface is impossible due to a complete coverage of the electrode surface, Fig. 2B. The DNA-biosensor response is only determined by interaction of the compound with the dsDNA in the film, without any electrochemical contribution from the drug.

\section{Conclusions}

Using ex situ MAC mode atomic force microscopy in air, we have been able to visualise directly the surface characteristics of the dsDNA films prepared at the HOPG electrode. It was found that different immobilization methodologies lead to structural changes on the DNA-biosensor surface and consequently different sensor response.

\section{Acknowledgements}

Financial support from Fundaç ão para a Ciência e Tecnologia (FCT), PhD grant PRAXIS XXI/BD/19728/99 (A.-M.C.), POCTI (co-financed by the European Community Fund FEDER), ICEMS (Research Unit 103) and European Projects QLK3-2000-01311 and HPRN-CT-200200186 are gratefully acknowledged.

\section{References}

[1] A.M. Oliveira Brett, S.H.P. Serrano, J.A.P. Piedade, Electrochemistry of DNA, in: R.G. Compton, G. Hancock (Eds.), Comprehensive Chemical Kinetics, Applications of Kinetic Modelling 37, Elsevier, Oxford, UK, 1999, pp. 91-119. Chapter 3.

[2] E. Palecek, Past, present and future of nucleic acids electrochemistry, Talanta 56 (2002) 809-819.

[3] A.M. Oliveira Brett, T.A. Macedo, D. Raimundo, M.H. Marques, S.H.P. Serrano, Voltammetric behaviour of mitoxandrone at a DNAbiosensor, Biosens. Bioelectron. 13 (1998) 861-867.

[4] A.M. Oliveira-Brett, M. Vivan, I.R. Fernandes, J.A.P. Piedade, Electrochemical detection of in situ adriamycin oxidative damage to DNA, Talanta 56 (2002) 959-970.

[5] M. Mascini, I. Palchetti, G. Marrazza, DNA electrochemical biosensors, Fresenius' J. Anal. Chem. 369 (2001) 15-22.

[6] W. Han, S.M. Lindsay, T.W. Jing, A magnetically-driven oscillating probe microscope for operation in liquids, Appl. Phys. Lett. 69 (1996) 4111-4113.

[7] W. Saenger, Principles of nucleic acid structure, in: Ch.R. Cantor (Ed.), Springer-Verlag, New York, 1984.

[8] B.P. Belotserkovskii, B.H. Johnston, Denaturation and association of DNA sequences by certain polypropylene surfaces, Anal. Biochem. 251 (1997) 251-262. 